\title{
Knidos als Zentrum der frühen wissenschaftlichen Medizin im antiken Griechenland
}

von Jutta Kollesch*

\section{ZuSAMMENFASSUNG}

Knidos und Kos, benachbart und auch durch das Arztegeschlecht der Asklepiaden verwandt, bilden im 5. Jahrhundert v. Chr. eine vielfältige, aber einheitliche Heilkunde aus. Der Stand der Quellen erlaubt es nicht, wie das oft getan wird, einen prinzipiellen Unterschied zwischen knidischer und koischer Medizin festzustellen. Die knidischen Ärzte Euryphon, Herodikos und Ktesias werden vorgestellt.

Jedermann weiß es oder kann es in den einschlägigen Medizingeschichten und Untersuchungen zur hippokratischen Medizin nachlesen, daß in der Frühzeit der wissenschaftlichen Medizin in Griechenland im ägäischen Raum auf der Insel Kos und in der in unmittelbarer Nähe auf dem Festland von Kleinasien gelegenen Stadt Knidos zwei Ärzteschulen mit deutlich voneinander abzugrenzenden medizinischen Lehren existierten und daß die uns erhaltene Sammlung hippokratischer Schriften neben den Werken koischer Ärzte auch Texte knidischen Ursprungs enthält, die als solche identifiziert und demzufolge als Quellenmaterial für die Rekonstruktion der knidischen Medizin benutzt werden können. Was man indessen nicht weiß oder zumindest nur bereit ist zur Kenntnis zu nehmen, ohne daraus die notwendigen Konsequenzen zu ziehen, ist der bedauerliche Tatbestand, daß die antiken Zeugnisse zur knidischen Medizin nicht nur der Zahl nach gering, sondern auch von der sachlichen Aussage her nicht allzu ergiebig sind, so daß sie bei unvoreingenommener Betrachtung weder für die Bestimmung dessen, worin die grundlegenden Unterschiede zwischen der knidischen und der koischen Medizin bestanden haben, noch für die Zuordnung bestimmter Schriften oder Schriftengruppen aus dem Corpus Hippocraticum an die knidische Schule eine gesicherte Grundlage bieten.

*Gastvorlesung, gehalten am 3. Mai 1988 an der Universität Zürich. 
Ein sehr instruktives Beispiel hierfür sind die beiden jüngsten monographischen Untersuchungen zur knidischen Medizin von Hermann Grensemann und Jacques Jouanna. ${ }^{1}$ Grensemann räumt zwar expressis verbis ein, daß die Auskünfte, die uns die von ihm zu Beginn seines Buches zusammengestellten Testimonien und Fragmente über die knidische Ärzteschule geben, sehr dürftig sind. ${ }^{2}$ Beide Autoren halten aber daran fest, daß es in Verbindung mit eben diesen antiken Nachrichten auf dem Umweg über das Corpus Hippocraticum möglich sei, «die Medizin der frühen Knidier», wie Grensemann es formuliert ${ }^{3}$, «... auf recht breiter Textbasis lesbar zu machen und damit einen Einblick zu tun in eine sehr frühe Form griechischer Medizin». Wenn man einerseits berücksichtigt, daß diese «sehr frühe Form griechischer Medizin» nach Auffassung von Grensemann der altägyptischen Heilkunde näher gestanden habe als der koischen, der er im Unterschied zu der von ihm als archaisch bezeichneten Medizin der knidischen Ärzte eine «völlig neue Denkart» bescheinigt ${ }^{4}$, und wenn man sich andererseits klarmacht, daß sich die Merkmale, die das Wesen der hippokratischen Heilkunde bestimmen, auch in den als knidisch verdächtigen Texten des Corpus Hippocraticum finden, so dürfte das allein schon genügen, um das Verfahren, knidisches Lehrgut mit Hilfe der Schriften der hippokratischen Sammlung zu erschließen, als fragwürdig erscheinen zu lassen.

Bevor wir auf den angeblichen Gegensatz zwischen der koischen und knidischen Medizin zu sprechen kommen, den Wesley D. Smith nicht ganz zu Unrecht vor nicht allzu langer Zeit als «figment of the scholarly imagination ${ }^{5}$ bezeichnet hat, erscheint es aus den genannten Gründen angebracht, ein unvoreingenommen-kritisches Bild der knidischen Medizin zu zeichnen, wie es sich uns aus den antiken Zeugnissen unterschiedlicher Provenienz darstellt, und auf solche Weise den sicheren Ausgangspunkt für die weitere Interpretation zu gewinnen.

Was die ägäische Insel Kos und die an der Westspitze der Chersones von Karien gelegene Stadt Knidos miteinander verband, war nicht nur die geographische Lage. Beide gehörten zum Siedlungsgebiet der Dorer, die im 11. Jh. v. u.Z. als letzter griechischer Stamm nach Griechenland einwanderten, bis zur Südspitze der Peloponnes vordrangen und von dort aus mehrere Inseln im Süden der Ägäis, unter ihnen auch Kos, und den südlichen Teil der Westküste Kleinasiens besiedelten. Nach Herodot (I 174 u. VII 99) stammten die Bewohner von Knidos aus Sparta und die der Insel Kos aus Epidauros. Über die ethnische Herkunft hinaus waren gewisse Berührungspunkte zwischen Kos und Knidos, zumindest in archaischer Zeit, durch die 
Mitgliedschaft beider in der dorischen Hexapolis gegeben, einem Bund, der, so ebenfalls Herodot (I 144), von den sechs dorischen Siedlungen Kos, Knidos, Halikarnaß, Kameiros, Ialysos und Lindos zum Zwecke der gemeinsamen Verehrung des Apollon Triopios, dessen Tempel sich in der Nähe von Knidos befand, geschlossen worden war.

Für die medizinische Tradition von Kos und Knidos ist es jedoch bedeutsam, daß es sowohl in Knidos als auch auf Kos Familien aus dem Geschlecht der Asklepiaden gegeben hat, einem angesehenen Adelsgeschlecht, in dem die Ausübung der ärztlichen Tätigkeit offenbar zur Tradition geworden war, so daß seine Angehörigen dies zum Anlaß nehmen konnten, ihren Stammbaum auf den Heilgott Asklepios zurückzuführen, und zwar über dessen Sohn Podaleirios, wie die spätere Überlieferung zu berichten weiß. Podaleirios soll sich bei seiner Rückkehr aus dem Trojanischen Krieg in Karien niedergelassen und dort, nachdem er die karische Königstochter geheiratet hatte, eine Stadt namens Syrnos gegründet haben, von wo seine Nachkommen, die Asklepiaden, nach Knidos und auf die Insel Kos gelangt seien. ${ }^{6}$ Der Zusammenhalt der koischen und der knidischen Asklepiadenfamilien scheint zumindest bis in das 4.Jh. v.u.Z. recht eng gewesen zu sein. Das bezeugt eine in Delphi gefundene Inschrift aus der ersten Hälfte des 4 . Jh. v. u. Z. ${ }^{7}$ mit einem von der Gemeinschaft, dem xoıvóv, der Asklepiaden aus Kos und Knidos gefaßten Beschluß, mit dem den Nachkommen des Asklepios in männlicher Linie bestimmte Privilegien in Delphi, nämlich der Zugang zum Orakel und das Darbringen von Opfern, garantiert werden sollten. Da zum einen davon auszugehen ist, daß nicht alle Asklepiaden in männlicher Linie auch Ärzte waren, und zum anderen in diesem Dokument der Kreis der Asklepiaden, die in den Genuß der genannten Privilegien kommen sollten, nicht von der Ausübung des ärztlichen Berufes, sondern von der männlichen Deszendenz abhängig gemacht wurde, wird man wohl mit einiger Sicherheit annehmen dürfen, daß es sich bei der inschriftlich erwähnten Gemeinschaft der Asklepiaden von Kos und Knidos um eine Vereinigung der ihrer Abstammung nach echten Asklepiaden im Unterschied zu den Familienangehörigen handelte, die mütterlicherseits aus dem Asklepiadengeschlecht stammten.

Wenn man dieser Inschrift unseres Erachtens mithin auch nicht entnehmen kann, daß sich die Asklepiaden von Kos und Knidos in einem gemeinsamen Berufsverband zusammengeschlossen hatten ${ }^{8}$, so ist sie doch ein untrüglicher Beweis dafür, daß zu Beginn des 4.Jh. v.u.Z., d.h. noch zu Lebzeiten des Hippokrates, bestimmte Bindungen zwischen den koischen 
und knidischen Asklepiadenfamilien bestanden haben, die es als gesichert erscheinen lassen, daß auch auf medizinischem Gebiet, dem bevorzugten Wirkungsfeld der Asklepiaden, eine Kommunikation zwischen Kos und Knidos stattgefunden hat. Dieser Umstand ist für die Bestimmung des Verhältnisses zwischen koischer und knidischer Medizin von größter Wichtigkeit, ist doch damit zum erstenmal auch ein äußeres Kriterium dafür gegeben, daß eine strikte Trennung zwischen den Lehrinhalten der koischen Medizin und denen der knidischen Heilkunde weder gerechtfertigt noch möglich ist. ${ }^{9}$

In welche Zeit die historischen Anfänge der von den Asklepiadenfamilien getragenen medizinischen Traditionen in Knidos und auf Kos fallen, wissen wir nicht. Ebensowenig läßt sich mit Sicherheit entscheiden, ob Kos oder Knidos die Priorität als Pflegestätte der Medizin gebührt. Möglicherweise entspricht es jedoch den historischen Gegebenheiten, wenn sich Knidos auf Grund der namentlich überlieferten Arztpersönlichkeiten und der, allerdings nur dem Titel nach bekannten, literarischen Produktion für uns als die ältere von den beiden Ärzteschulen darstellt.

Als ältestes Zeugnis der knidischen Ärzteschule gelten die «Knidischen Sentenzen». Sie werden von dem Verfasser der hippokratischen Schrift De diaeta acutorum und in Galens Kommentar zu diesem Text als ein von mehreren Autoren verfaßtes Werk bezeichnet, von dem in der zweiten Hälfte des 5.Jh. v.u.Z., der Entstehungszeit von De diaeta acutorum, eine als später überarbeitet bezeichnete Fassung existierte ${ }^{10}$, so daß man annehmen kann, da $\beta$ die Originalfassung des Textes spätestens aus der ersten Hälfte des 5. Jh. stammt. In seinem Konmentar zum 6. Buch der hippokratischen «Epidemien» (In Hipp. Epid. VI comm. I 29: CMG V 10,2,2, S.54,1-6) erwähnt Galen jedoch, daß die «Knidischen Sentenzen» dem knidischen Arzt Euryphon, der als älterer Zeitgenosse des Hippokrates zu Beginn des 5. Jh. geboren sein dürfte, als Verfasser zugewiesen wurden; da Galen selbst sich aber von dieser Zuschreibung eindeutig distanziert, steht die in der modernen Forschung auf Grund dieser Galenstelle allgemein akzeptierte These, daß die «Knidischen Sentenzen», sei es in ihrer ursprünglichen, sei es in ihrer überarbeiteten Fassung, als Werk des Euryphon zu gelten haben, auf recht unsicherem Boden. ${ }^{11}$

Die kollektive Verfasserschaft der «Knidischen Sentenzen» legt die Annahme nahe, daß ihnen schriftliche Aufzeichnungen verschiedener Ärzte zugrunde lagen, die dem elementaren Bedürfnis entsprachen, die im Umgang mit Krankheiten und deren Therapie gemachten Erfahrungen und 
Beobachtungen zu sammeln und als Arbeitsmaterial nutzbar zu machen. Sie enthielten Symptombeschreibungen, gelegentlich aber auch Angaben zum Verlauf bzw. Ausgang der Krankheiten und Therapiehinweise ${ }^{12}$ und sind, wie man sich vorstellen könnte, der Übersichtlichkeit halber, insofern sie auf Grund der Angabe der Krankheitsbezeichnung einer bestimmten Krankheit zugeordnet werden konnten, jeweils zu einem Komplex zusammengefaßt worden. Galen berichtet in seinem Kommentar zu De diaeta acutorum (In Hipp. De victu acut. comm. I 7: CMG V 9,1, S.121,21-122,3), daß die knidischen Ärzte sieben Krankheiten der Galle, zwölf Blasenkrankheiten, vier Nierenkrankheiten, vier Arten von Harnverhaltung, drei Arten von Tetanus, vier Arten von Gelbsucht und drei Arten von Schwindsucht gezählt hätten. An diesem Verfahren übt der Autor von De diaeta acutorum Kritik ${ }^{13}$, wobei er sich einerseits gegen das Unterfangen wendet, die in Abhängigkeit vom individuellen Krankheitsgeschehen unterschiedlichen Erscheinungsformen einer speziellen Krankheit mit genauen Zahlen angeben zu wollen, und andererseits die Unübersichtlichkeit einer derartigen Zählung hervorhebt, die er darin begründet sieht, daß die individuell verschiedenen Spielarten ein und derselben Krankheit dann, wenn sie einmal mit unterschiedlichen Bezeichnungen, d.h. jeweils mit einer anderen Nummer, versehen sind, als voneinander verschiedene Erkrankungen angesehen wurden. Da er im Zusammenhang mit seinem Vorwurf nur von «einigen» Ärzten spricht, muß die Frage offenbleiben, ob die Krankheitsbeschreibungen innerhalb eines Komplexes, soweit sie sich in der Angabe der Symptome und in der Darstellung des Krankheitsverlaufs voneinander unterschieden, bereits in den «Knidischen Sentenzen» als verschiedene Arten ein und derselben Krankheit gekennzeichnet und durchgezählt waren oder ob dieses Verfahren erst in späteren Texten entwickelt wurde.

Die neuerlichen Bemühungen von Grensemann und Jouanna, nachzuweisen, daß die einzelne Sentenz, d.h. die Beschreibung einer einzelnen Krankheit, ähnlich wie bei den aus den altägyptischen medizinischen Texten bekannten Diagnosen unter Verwendung stereotyper sprachlicher Wendungen jeweils nach einem festen Schema aufgebaut war, als dessen obligatorische Bestandteile von ihnen Titel, Symptombeschreibung, Prognose und Therapie, allerdings in unterschiedlicher Kombination und Reihenfolge, genannt werden ${ }^{14}$, sind nach unserem Dafürhalten bereits vom Ansatz her verfehlt. Denn von den «Knidischen Sentenzen» sind lediglich zwei kurze Bruchstücke von eineinhalb und zweieinhalb Zeilen wörtlich überliefert, die keinerlei Aussagen über Gesamtumfang und Aufbau auch 
nur einer einzelnen Sentenz zulassen, so daß letzten Endes zwei Kapitel aus den im Corpus Hippocraticum enthaltenen Schriften De morbis II (Kap. 68) und De muliebribus II (Kap. $144=$ De nat.mul.5) als Modell zur Rekonstruktion der «Knidischen Sentenzen» dienen müssen, d.h. Texte, deren Herkunft aus den «Sentenzen» jedoch eigentlich erst zu beweisen war. Das bedeutet aber, daß wir auch die auf dieser Voraussetzung basierende Rekonstruktion der «Knidischen Sentenzen» nicht als gesicherte Grundlage für eine Darstellung der durch diese Texte des Corpus Hippocraticum repräsentierten ältesten Stufe der knidischen Medizin akzeptieren können. ${ }^{15}$

Was wir auf Grund der wenigen authentischen Zeugnisse ${ }^{16}$ von dieser Medizin sagen können, ist lediglich, daß ihre Vertreter Krankheitsbezeich-

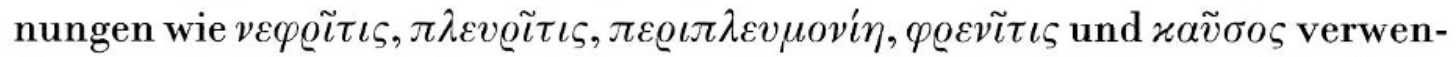
deten und daß zumindest einige von ihnen die Krankheiten an Hand der Krankheitsnamen zu klassifizieren versuchten, daß sie ihr Hauptaugenmerk auf die Krankheitszeichen richteten, wobei sie neben Schmerzempfindungen in den jeweils betroffenen Körperteilen auch krankhafte Veränderungen an den Körperausscheidungen registrierten, daß sie darüber hinaus auch dem Krankheitsverlauf eine gewisse Bedeutung beimaßen und daß sie sich schließlich in ihren therapeutischen Verordnungen auf relativ wenige Mittel wie Abführmittel und je nach Jahreszeit Molke oder Milch beschränkten und bei der Vergabe der Mittel mehr oder weniger schematisch vorgingen - ein Verfahren, das offenbar nicht nur durch die geringe Anzahl der zur Verfügung stehenden Heilmittel, sondern auch durch den Mangel an therapeutischer Erfahrung bedingt war, der es noch nicht erlaubte, die therapeutischen Maßnahmen entsprechend den verschiedenen Krankheiten und deren Verlauf zu differenzieren.

Wie bereits erwähnt, hatte es nach Aussage Galens eine antike Tradition gegeben, welche die «Knidischen Sentenzen» dem knidischen Arzt Euryphon als Verfasser zugewiesen hat. Diese, wie wir glauben, nachträglich erfolgte Zuweisung dürfte sich aus dem Bestreben heraus erklären, die älteste knidische medizinische Schrift mit dem Namen des ältesten und zugleich berühmtesten Arztes von Knidos zu verbinden. So ist Euryphon in ähnlicher Weise ebenfalls schon in der Antike neben anderen alten Ärzten auch als möglicher Verfasser von hippokratischen Schriften, z. B. von De diaeta und De septimanis, namhaft gemacht worden. ${ }^{17}$ Anspruch auf Glaubwürdigkeit können diese Zuweisungen indessen nicht erheben, so daß uns für die Darstellung seiner Lehren nur die spärlichen mit seinem Namen verbundenen Nachrichten bei späteren Autoren zur Verfügung stehen. 
Das älteste Zeugnis ist die in der Medizingeschichte des Aristotelesschülers Menon überlieferte Krankheitsätiologie des Euryphon. ${ }^{18}$ Danach soll der Knidier die Rückstände von unverdauter Nahrung ( $\pi \varepsilon \varrho \sigma \sigma \omega ́ \omega \mu \alpha \tau \alpha)$, die vom Bauch zum Kopf aufsteigen, als Krankheitsursache genannt haben, wohingegen ihm der dünne und reine Leib als Zeichen einer normal funktionierenden Verdauung galt. Da wir jedoch von Galen wissen, daß Aristoteles der erste antike Autor gewesen ist, der die Nahrungsrückstände im Körper mit dem Ausdruck $\pi \varepsilon \varrho \iota \tau \tau \omega ́ \omega \alpha \tau \alpha$ bezeichnet hat ${ }^{19}$, darf als sicher angenommen werden, daß Menon den Begriff der $\pi \varepsilon \varrho \iota \sigma \sigma \omega ́ \mu \alpha \tau \alpha$ aus seiner eigenen Vorstellungswelt auf die Krankheitsätiologie des Euryphon übertragen hat. Wie der Knidier selbst das, was seiner Meinung nach infolge von Verdauungsstörung zum Kopf aufsteigt, bezeichnet hat, läßt sich nicht mehr ausmachen; und ebensowenig läßt sich entscheiden, ob und inwiefern er auch Wärme und Kälte sowie Anstrengung und Überfüllung als Krankheitsursachen berücksichtigt hat, denn bei diesem Hinweis Galens handelt es sich um ein Sammelreferat, in dem neben dem Knidier noch weitere vier Ärzte genannt sind ${ }^{20}$.

Daß Euryphon es sich ebenso wie die Verfasser der «Knidischen Sentenzen» zur Aufgabe gemacht hatte, die Krankheitszeichen sorgfältig zu beobachten, beweist seine Beschreibung der beim bläulichen Fieber ( $\pi \varepsilon \lambda \iota \delta v o ̀ \varsigma \pi v \varrho \varepsilon \tau o ́ \varsigma)$ auftretenden Symptome, die Galen als wörtliches Zitat überliefert hat. ${ }^{21}$ Der Wortlaut dieses Zitats stimmt weitgehend mit der Beschreibung dieses Fiebers in der hippokratischen Schrift De morbis II ${ }^{22}$ überein. Allerdings erscheint es uns entgegen der communis opinio nicht gerechtfertigt, von der Identität dieser beiden Texte zu sprechen; denn zum einen finden sich in den Texten mehrere Abweichungen, die nach unserem Dafürhalten so gravierend sind, daß sie kaum noch als überlieferungsbedingte Varianten ein und desselben Textes erklärt werden können, und zum anderen sind eben diese beiden Textstücke von Galen nebeneinander als zwei in ihrer sachlichen Aussage übereinstimmende, aber in ihrer Herkunft voneinander unabhängige Textzeugnisse zitiert worden, was zumindest ausschließt, daß Galen diese Texte für identisch gehalten hat. ${ }^{23}$ Dennoch ist die Ähnlichkeit der beiden Krankheitsbeschreibungen insofern aufschlußreich, als sie zu erkennen gibt, daß die Beobachtungen, Erfahrungen und Erkenntnisse, welche die knidischen Ärzte und speziell Euryphon in ihren nosologischen Schriften zusammengetragen hatten, von den nachfolgenden Ärztegenerationen als brauchbares Arbeitsmaterial akzeptiert und in Abhängigkeit von dem Wissen und den Ambitionen der einzelnen 
Ärzte in mehr oder weniger stark modifizierter Form weitertradiert worden sind.

Aus der Nosologie des Euryphon erfahren wir weiterhin, daß er unter Pleuritis eine Erkrankung der Lunge verstanden hat und daß er zwei Arten von Blutfluß annahm. ${ }^{24}$ Allerdings wird man die Behauptung des spätantiken Autors Caelius Aurelianus, der knidische Arzt habe Blutungen aus den Venen (venae) und Arterien (arteriae) gekannt, als eine Fehlinterpretation des zugrunde liegenden Euryphontextes anzusehen haben. ${ }^{25}$ Denn es kann

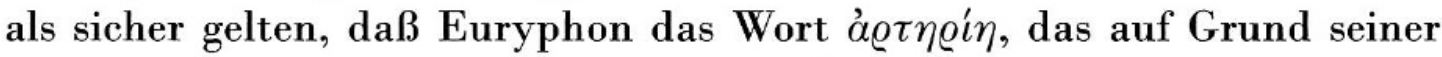
Mehrdeutigkeit zu dem Mißverständnis geführt haben dürfte, entsprechend dem frühen griechischen Sprachgebrauch zur Bezeichnung der Luftröhre verwendet hat und das Wort $\varphi \lambda \hat{\varepsilon} \beta \varepsilon \varsigma$ als neutralen Ausdruck für die Adern gebrauchte, so daß also, wenn bei ihm außer von Blutflüssen aus den Adern

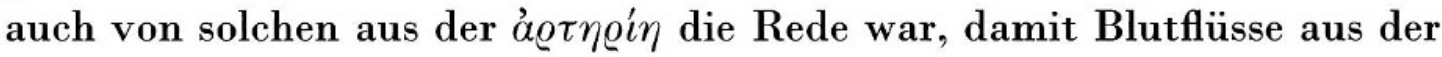
Luftröhre gemeint waren. So erweist sich auch die in der Forschung gelegentlich vertretene Auffassung, Euryphon habe bereits zwischen Venen und Arterien unterschieden ${ }^{26}$, als hinfällig.

Von der Therapie des Euryphon ist überliefert, daß er bei Hydrops ebenso wie sein Landsmann Herodikos Abführmittel verabreichte, nach dem Essen Erbrechen provozierte, Bedampfungen vornahm und das Schlagen der geschwollenen Körperteile mit aufgeblähten Rindsblasen empfahl, daß er Muttermilch, direkt an der Brust der Frau getrunken, für das beste Heilmittel gegen Schwindsucht hielt, daß er den Aderlaß als Therapeuticum anwandte, und endlich, daß er sich über den Gebrauch der Pharmaka geäußert und als einer der ersten mit der Darstellung von Ersatzmitteln beschäftigt haben soll. ${ }^{27}$

Zu den medizinischen Gebieten, auf denen Euryphon tätig gewesen ist, gehören auch die Gynäkologie und Geburtshilfe. Max Wellmanns Annahme, der Knidier habe eine speziell diesem Gegenstand gewidmete Schrift mit

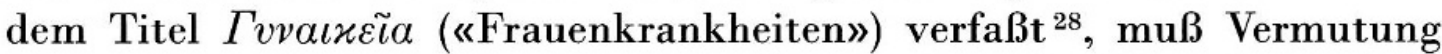
bleiben, da in den in Frage kommenden Testimonien jeder Hinweis auf einen Schriftentitel fehlt. Aus der geburtshilflich-gynäkologischen Praxis des Euryphon berichtet Soran von Ephesos (Gyn. I 35,3; IV 14,2: CMG IV, S. 24,24 f.; 144,23-27), daß dieser zum Nachweis von Empfängnisfähigkeit an den auf einem Gebärstuhl sitzenden Frauen Gebärmutterräucherungen mit stark riechenden Substanzen vorgenommen habe und daß er bei der Verhaltung der Nachgeburt harntreibende Getränke, bluttreibende Einlagen und das Schütteln der Frau mit Hilfe einer Leiter empfahl, an die sie 
zuvor angebunden wurde. Euryphons Behandlung des prolabierten Uterus, bei der die Patientin nach der Darstellung des Soran (Gyn. IV 36, 7: CMG IV, S. 149,9-13) zunächst einen Tag und eine Nacht lang mit den Füßen an einer Leiter aufgehängt wurde und danach auf dem Rücken liegen und kalten Gerstenschleim als Nahrung zu sich nehmen mußte, ist von dem Ephesier (ebd., Z.13-15) mit dem Hinweis darauf, daß das Hängen an der Leiter unerträglich sei, daß die verordnete Nahrung eine blähende Wirkung habe und daß die Berechnung der für die Einleitung der therapeutischen Maßnahmen günstigen Tage nicht den Regeln der Kunst entspreche, gerügt worden. Als außergewöhnlich wurde in der späteren doxographischen Tradition auch die der landläufigen Meinung widersprechende Ansicht des Euryphon vermerkt, daß die Siebenmonatskinder nicht lebensfähig seien. ${ }^{29}$ Daß Euryphon bereits die Ovarien und die Eileiter gekannt habe und diese in den Blasenhals einmünden ließ, wie Wellmann schreibt ${ }^{30}$, läßt sich nicht stichhaltig beweisen, da an der von ihm angeführten Stelle aus Galens Schrift De uteri dissectione (9,5: CMG V 2,1, S.48,22-50,7) ganz offensichtlich eine unzulässige Verallgemeinerung vorliegt, wenn der Pergamener die von Herophilos stammende Beschreibung der weiblichen Keimdrüsen und der weiblichen «Samengänge» auch für Aristoteles und unseren knidischen Arzt in Anspruch nimmt. ${ }^{31}$ Angesichts dieser Situation verbietet es sich auch, daraus irgendwelche Schlußfolgerungen hinsichtlich der anatomischen Tätigkeit des Euryphon zu ziehen.

Zu den alten knidischen Ärzten, die uns namentlich bekannt sind, zählt auch Herodikos. Er wird zwar nur in der Medizingeschichte des Menon (Anon.Lond. IV $40 \mathrm{f}$.) als Knidier bezeichnet, ist aber mit großer Wahrscheinlichkeit identisch mit dem Herodikos, den Galen neben Euryphon als Vertreter der Auffassung nennt, daß Muttermilch das beste Heilmittel bei Schwindsucht sei ${ }^{32}$, und dessen Therapie des Hydrops bei Caelius Aurelianus und Aetios von Amida überliefert ist. ${ }^{33}$ Nicht gerechtfertigt erscheint dagegen der Versuch Grensemanns, Herodikos von Selymbria mit dem Knidier zu identifizieren. ${ }^{34}$ Erstens gibt es keinerlei Anhaltspunkte dafür, daß Herodikos von Selymbria, eine uns aus anderen Zeugnissen als Paidotribe und Arzt bekannte Persönlichkeit des 5. Jh. v.u.Z., sich auch in Knidos aufgehalten hat, und zweitens widerspricht dem auch der Umstand, daß in der Medizingeschichte des Menon nach der Erwähnung des Herodikos von Knidos wenig später (Anon.Lond.IX 20) noch ein weiterer Herodikos genannt wird. Wenn es nun auch auf Grund der starken Beschädigung des Papyrus gerade an dieser Stelle unsicher bleiben muß, ob hier von Herodikos 
von Selymbria die Rede ist, wie der Herausgeber Hermann Diels vermutet hat, so bleibt doch die Tatsache bestehen, daß im 4.Jh. v.u.Z., als Menon seine Medizingeschichte schrieb, zwei Ärzte dieses Namens bekannt waren, denen, wie die Menonische Darstellung beweist, unterschiedliche Krankheitsätiologien zugeschrieben wurden.

Sowohl die Tatsache, daß Menon die Krankheitsätiologie des Herodikos nach der des Euryphon anführt, wie auch der Umstand, daß die Ätiologie des Herodikos differenzierter ist als die des Euryphon, sprechen dafür, daß Herodikos der jüngere von den beiden gewesen ist. ${ }^{35}$ Wenn wir andererseits voraussetzen dürfen, daß er älter war als Hippokrates, kann der Altersunterschied jedoch nicht sehr groß gewesen sein, so daß wir mit seiner Hauptschaffensperiode etwa in die Mitte des 5. Jh. v.u.Z. kommen.

Nach dem aus der Medizingeschichte des Menon stammenden Bericht über die Krankheitsätiologie des Herodikos (Anon. Lond. IV 40-V 34) hat dieser ebenso wie Euryphon die unverdauten Nahrungsrückstände im Körper für das Entstehen von Krankheiten verantwortlich gemacht. Diese Etikettierung trifft insofern zu, als Herodikos die den Krankheitsprozeß einleitende Ursache in der durch Mangel an körperlicher Bewegung bedingten ungenügenden Verarbeitung der aufgenommenen Nahrung gegeben sah, aus deren unverdauten Rückständen zwei Arten von Flüssigkeiten entstehen, eine scharfe und eine bittere, die ihrerseits die Krankheiten hervorrufen. Bemerkenswert ist, daß Herodikos in seiner Krankheitsätiologie darüber hinaus auch das Zustandekommen der unterschiedlichen Arten von Krankheiten zu erklären versuchte. Die Kriterien, die er in diesem Zusammenhang nennt, sind erstens das Überwiegen einer der beiden Flüssigkeiten, zweitens die bei den Flüssigkeiten auftretende unterschiedliche Intensität der Schärfe bzw. Bitterkeit, drittens, ob ein bestimmter Körperteil, z. B. der Kopf, entweder von der bitteren oder von der scharfen Flüssigkeit befallen wird, und viertens, welcher Körperteil es ist, zu dem die Flüssigkeiten gelangen. Diese differenzierende Krankheitsätiologie des Herodikos enthält bereits deutliche Ansätze sowohl einer Organlehre als auch einer Säftetheorie, die das Bemühen der frühen knidischen Ärzte erkennen lassen, das Krankheitsgeschehen in seiner Vielfalt zu erfassen und theoretisch zu begründen.

Aus Knidos stammte auch der Arzt Ktesias ${ }^{36}$, der, wenn wir Galen (In Hipp. De artic. comm. IV 40: XVIII A, S.731,7f. Kühn) Glauben schenken dürfen, seiner Herkunft nach ein Asklepiade war. Als Kriegsgefangener nach Persien gekommen, war er längere Zeit Leibarzt der persischen Königin 
Parysatis und später auch ihres Sohnes Artaxerxes II. Mnemon (404-358), den er ärztlich versorgte, als dieser von seinem Bruder Kyros in der Schlacht von Kunaxa im Jahre 401 v.u.Z. verwundet worden war. Als Ktesias 398/397 als Gesandter nach Sparta geschickt wurde, nutzte er diese Gelegenheit zur Rückkehr in seine Heimat. Mit seiner «Geschichte Persiens» in 23 Büchern und seiner drei Bücher umfassenden Beschreibung Indiens, die beide nur in Fragmenten erhalten sind, hat er sich vor allem als Historiker einen Namen gemacht. Aus den medizinischen Schriften des Ktesias, über deren Zahl und Inhalt nichts Näheres bekannt ist, hat Oreibasios (Coll. med. rel. VIII 8: CMG VI 1,1, S. 261,19-25) ein wörtliches Zitat über die Anwendung des Helleboros (Nieswurz) überliefert. Ktesias schreibt dort, daß dieses Mittel zur Zeit seines Vaters und seines Großvaters von keinem Arzt verordnet wurde, da man seine Mischung nicht kannte und auch nicht wußte, in welcher Dosierung es zu verabreichen sei. Zumindest hätte derjenige, der einen Patienten der mit dem Einnehmen der Nieswurz verbundenen Gefahr aussetzte, diesen zuvor sein Testament machen lassen, denn die Einnahme hätte in vielen Fällen zum Erstickungstod geführt, und nur wenige seien mit dem Leben davongekommen. Jetzt, d.h. zur Zeit des Ktesias, scheine in dieser Frage jedoch größere Sicherheit zu herrschen. Dieses Zitat aus den Schriften des K tesias ist nicht nur insofern interessant für uns, als es deutlich werden läßt, daß im Verlauf des 5. Jh. v.u.Z. auch auf dem Gebiet der Pharmakologie bzw. der Pharmakotherapie ein gewisser Fortschritt erzielt worden ist, sondern vor allem deswegen, weil es bestimmte Schlüsse auf die medizinischen Vorstellungen des Ktesias zuläßt. Den Anhaltspunkt hierfür bilden die Begriffe der Mischung, der Krasis, wie es griechisch heißt, und der richtigen Dosierung, die für unseren Arzt die entscheidende Voraussetzung für eine erfolgreiche Anwendung des Helleboros darstellen. Was die Forderung nach der richtigen Dosierung des Mittels betrifft, so geht sie auf die durch sorgfältige Beobachtung gewonnene Erkenntnis zurück, daß einige Heilmittel, wenn sie in zu großer Menge verabreicht werden, auch schädlich wirken können, eine Erkenntnis, der wir auch in der zeitgenössischen hippokratischen Medizin begegnen. ${ }^{37}$ Und wenn zum anderen von der «Mischung der Nieswurz» die Rede ist, so ist unseres Erachtens damit nicht, wie Grensemann zu glauben scheint ${ }^{38}$, die Zusammensetzung der Nieswurz mit anderen Substanzen wie Wein oder Honig gemeint; die sprachliche Formulierung spricht vielmehr dafür, daß Ktesias unter der Krasis der Nieswurz eine in dieser Pflanze festzustellende Mischung von Qualitäten verstanden hat. $\mathrm{Ob}$ es sich nun aber bei diesen 
Qualitäten um Primärqualitäten (warm, feucht, kalt, trocken) oder Sekundärqualitäten (süß, salzig, bitter, sauer usw.) handelte, läßt sich an Hand der kurzen Bemerkung nicht entscheiden. Fest steht jedoch, daß Ktesias' Vorstellung von der Krasis der pflanzlichen Heilmittel auf der Säftelehre fußt, wie dies in ähnlicher Weise auch an den ersten Ansätzen zu einer theoretischen Erfassung der Arzneimittelwirkungen in den Schriften des Corpus Hippocraticum aus der Zeit um die Wende vom 5. zum 4. Jh. ablesbar ist. ${ }^{39}$

Ein weiterer Hinweis auf die medizinische Schriftstellerei des Ktesias findet sich bei Galen, der berichtet, daß der Knidier der erste gewesen sei, der Hippokrates wegen der Einrenkung des luxierten Hüftgelenks Vorwürfe gemacht habe, da dieses Gelenk doch sofort wieder ausrenke. ${ }^{40}$ Wenn es auch fraglich bleiben muß, ob Ktesias seinen koischen Kollegen tatsächlich namentlich genannt hat oder ob seine polemische Äußerung erst später auf Hippokrates bezogen wurde und ob sich die Kritik des Ktesias speziell gegen die der Hüftgelenksluxation gewidmeten Ausführungen in der im Corpus Hippocraticum überlieferten Schrift De articulis ${ }^{41}$ richtete, so wird man doch auf jeden Fall sagen können, daß Ktesias zu den Ärzten gehörte, die eine erfolgreiche Behandlung des luxierten Hüftgelenks für unmöglich hielten.

Von dem speziellen Interesse des Arztes zeugen auch die medizinischen Details, die Ktesias seinen Lesern in seinen historischen Schriften mitteilt. So berichtet er z. B. in der «Geschichte Persiens» ${ }^{42}$, daß Kyros, als er in der Schlacht von Kunaxa von einem Speer an der Schläfe getroffen wurde, infolge des starken Blutverlustes einen Schwindelanfall erlitt und bewußtlos wurde und danach nur mühsam wieder zu sich kam. Als Kyros bald darauf bei einem erneuten Anschlag wiederum von einem Speer getroffen wurde, sei die Ader in der Kniekehle aufgeplatzt, Kyros sei, als er niederstürzte, mit der verletzten Schläfe auf einen Stein aufgeprallt und habe so den Tod gefunden. Auf das medizinische Interesse des Ktesias dürfte es auch zurückzuführen sein, wenn er es in seiner Beschreibung Indiens für erwähnenswert hielt, daß die Inder weder an Kopfschmerzen noch an Augenkrankheiten, Zahnschmerzen, Mundgeschwüren und Mundfäule litten. ${ }^{43}$

Gewisse Aufschlüsse über die Verhaltensweisen des Arztes gegenüber seinen Patienten zur Zeit des Ktesias können wir aus dem Bericht des Knidiers über den unrühmlichen Tod des koischen Arztes Apollonides gewinnen ${ }^{44}$, der unter Artaxerxes I. (464-424) Leibarzt am persischen Königshof war. Ktesias erzählt, Apollonides sei in Amytis, die Witwe des Satrapen Megabyzos und Schwester des Artaxerxes, verliebt gewesen und 
habe ihr, als sie nach seiner Diagnose an einem Gebärmutterleiden erkrankt war, aus therapeutischen Gründen Geschlechtsverkehr empfohlen. Er habe daraufhin Beziehungen $\mathrm{zu}$ ihr unterhalten, sich jedoch wieder von ihr getrennt, als sie immer schwächer wurde. Daraufhin habe Amytis ihre Mutter beauftragt, dafür zu sorgen, daß Apollonides bestraft würde, und nachdem diese dem König den Vorfall berichtet hatte, und zwar unter ausdrücklichem Hinweis darauf, daß Apollonides aus Hochmut Amytis verlassen habe, erhielt sie die Erlaubnis, die Bestrafung selbst vorzunehmen. Sie ließ Apollonides zunächst ins Gefängnis werfen und nach dem Tode ihrer Tochter lebendig begraben. Die Art und Weise, in der Ktesias diese Ereignisse dargestellt hat, läßt keinen Zweifel daran, daß Apollonides deswegen mit dem Tode bestraft wurde, weil Amytis sich an ihrem Liebhaber dafür rächen wollte, daß er sie verlassen hatte. Die Tatsache, daß Apollonides, selbst wenn wir ihm konzedieren, daß die von ihm empfohlene Therapie den medizinischen Vorstellungen seiner Zeit entsprach, seine Stellung als Arzt dazu ausgenutzt hat, mit der von ihm begehrten Frau Geschlechtsverkehr zu haben, scheint in diesem Zusammenhang weder für die Beteiligten noch für den Berichterstatter eine Rolle gespielt zu haben. Zumindest hat Ktesias auf jedes moralische Urteil über das Verhalten seines Kollegen verzichtet, so daß wir mit einer gewissen Berechtigung annehmen dürfen, daß der geschlechtlicke Umgang mit den Patienten, der nach den deontologischen Bestimmungen des sogenannten hippokratischen Eides unter die unehrenhaften Handlungen fällt, deren der Arzt sich zu enthalten hat ${ }^{45}$, weder von dem koischen Arzt Apollonides noch von dem Knidier als Verstoß gegen die ethischen Prinzipien ärztlichen Verhaltens betrachtet wurde. Diese Feststellung ist in Hinblick auf die Arzt-Patient-Beziehung in der Antike insofern interessant, als sie deutlich macht, daß es selbst für die zur ärztlichen Elite zählenden Ärzte aus Kos und Knidos keineswegs als selbstverständlich vorausgesetzt werden kann, daß sie sich in ihrem Auftreten gegenüber den Kranken an irgendwelche ethischen Normen gebunden fühlten oder gar die in dem eben genannten Eid ausgesprochene Forderung nach dem Schutz der persönlichen Integrität des Patienten zum obersten Grundsatz ihres ärztlichen Handelns erhoben hätten.

Der Vollständigkeit halber sei noch erwähnt, daß die uns bekannte Tradition der knidischen Ärzteschule bis in die Zeit um 300 v.u.Z. hinabreicht und daß als bekanntester Vertreter der späteren knidischen Medizin Chrysipp gilt, dessen Lehren sich bekanntlich Erasistratos zu eigen gemacht hat. Diese späte Tradition der knidischen Schule ist indessen für uns, die wir 
uns mit Knidos als dem Zentrum der frühen griechischen wissenschaftlichen Medizin beschäftigen wollten, belanglos. Von Belang ist jedoch noch die Beantwortung der Frage, inwieweit die alte knidische Medizin eine eigene Wertigkeit aufwies und ob grundsätzliche Unterschiede zu der Heilkunde der koischen Schule bestanden.

Wenn die von uns eingangs erwähnte inschriftliche Bezeugung der Gemeinschaft der koischen und knidischen Asklepiadenfamilien eine scharfe Trennung zwischen den theoretischen Auffassungen der Ärzte von Kos und Knidos in der ältesten Entwicklungsperiode nahezu mit Sicherheit ausschließt, so ist das Problem, das durch die Forschungsergebnisse von Grensemann und Jouanna aufgeworfen wurde, schwieriger zu lösen. ${ }^{46}$ Ich will keineswegs bestreiten, daß eine eingehende formale und sprachlichstilistische Analyse der nosologischen und gynäkologischen Texte des Corpus Hippocraticum, wie sie von ihnen vorgenommen wurde, wichtig ist, weil sie dazu beitragen kann, innere Widersprüche in diesen Texten zu lösen und ihr Verhältnis zueinander näher zu bestimmen. Unsere Bemerkungen und Einwände gegen den Versuch, die «Knidischen Sentenzen» zu rekonstruieren, zeigen indessen, daß wir keine Möglichkeit haben, mit ihrer Hilfe festzustellen, daß bestimmte Schriften des Corpus den «Sentenzen» formal und inhaltlich besonders nahestehen, und daraus ihren knidischen Ursprung abzuleiten. Solange dies aber nicht möglich ist, erscheint es auch nicht statthaft, die in ihnen vertretenen medizinischen Lehren insgesamt für die knidische Schule in Anspruch zu nehmen und sie als Ausdruck bestimmter Entwicklungstendenzen allein innerhalb dieser Schule zu werten. Nimmt man hinzu, daß, wie Robert Joly gezeigt hat ${ }^{47}$, in allen Schriften des Corpus die Grundannahmen der hippokratischen Humoralpathologie nachgewiesen werden können und daß somit sowohl in den koischen wie in den als knidisch verdächtigen Schriften im wesentlichen gleiche theoretische Auffassungen vertreten werden, so wird man nicht umhinkönnen, einzugestehen, daß die knidische Medizin für uns weiterhin das bleiben muß, was sie auch bislang schon war, nämlich ein untrennbarer und schwer abzugrenzender Bestandteil der griechischen wissenschaftlichen Heilkunde des 5. und 4. Jh., wie sie sich uns als Ganzes in der hippokratischen Schriftensammlung darstellt und wie sie sich im Gegensatz zu der vorgriechischen Heilkunde vor allem im Abstreifen von religiös-magischen Vorstellungen über den Ursprung der Krankheit sowie im Begreifen der Krankheit als eines sich in einem bestimmten Zeitraum abspielenden Prozesses äußert.

Wir werden, so glaube ich, der historischen Realität weit eher gerecht, 
wenn wir uns darum bemühen, die griechische Medizin aus der Zeit zwischen 450 und 350 als das Ergebnis einer ständigen wechselseitigen Beeinflussung zwischen Kos und Knidos zu begreifen und die unterschiedlichen Auffassungen in bestimmten Fragen als Vielfalt in der Einheit zu erklären, anstatt immer wieder nur von zwei rivalisierenden Schulen zu sprechen und um jeden Preis nach fundamentalen Unterschieden in den Lehrmeinungen der Ärzte von Kos und Knidos zu suchen.

\section{Anmerkungen}

1 H. Grensemann, Knidische Medizin. Teil I: Die Testimonien zur ältesten knidischen Lehre und Analysen knidischer Schriften im Corpus Hippocraticum, Berlin-New York 1975 (Ars Medica II 4,1); J.Jouanna, Hippocrate. Pour une archéologie de l'école de Cnide, Paris 1974.

2 H.Grensemann, a.a.O., S. 50.

3 Ebd., S. 51; vgl. J. Jouanna, a. a. O., S.3-6.

4 Siehe H.Grensemann, a. a.O., S. 50; 53; 201.

5 W.D.Smith, Galen on Coans versus Cnidians, Bull. of the Hist. of Medicine 47 (1973), S. 569 .

6 Siehe E.J.Edelstein u. L.Edelstein, Asclepius. A collection and interpretation of the testimonies, Baltimore 1945, Bd. II, S. 19 f. (Nachdruck New York 1975).

7 Publiziert bei J.Bousquet, Inscriptions de Delphes, Bull. de Correspondance Hellénique 80 (1956), S. 579 und Tafel X; zur Datierung s. ebd., S. 580 f.

8 So J.Bousquet, S. $582 \mathrm{f}$.

9 Vgl. F. Kudlien, Bemerkungen zu W. D.Smith's These über die knidische Ärzteschule, in: Corpus Hippocraticum. Actes du Colloque hippocratique de Mons (22-26 septembre 1975), hrsg. v. R.Joly, Mons 1977 (Editions Universitaires de Mons, Série Sciences Humaines IV), S. $102 \mathrm{f}$.

10 Hippokrates, De diaeta acut. 1. 3 (1): I, S. 109,2 f.; 110,3 Kühlewein = II, S.224,2 f.; 226,8 Littré; Galen, In Hipp. De victu acut. comm. I 1: C(orpus) M(edicorum) G(raecorum) V 9,1, S. 117,11 f.

11 Vgl. J.Kollesch, Die Stellung der knidischen Heilkunde in der wissenschaftlichen Medizin der Griechen, in: Corpus Hippocraticum (s.Anm.9), S.109, und W. D.Smith, a.a.O., S. 577 .

12 Siehe Hippokrates, De diaeta acut. 1. 2-3 (1): I, S. 109,2-5. 11-110,7 Kühlewein = II, S. 224,2-4. 9-226,11 Littré.

13 Siehe ebd. 3 (1): I, S. 110,7-13 Kühlewein = II, S.226,11-228,6 Littré; vgl. dazu

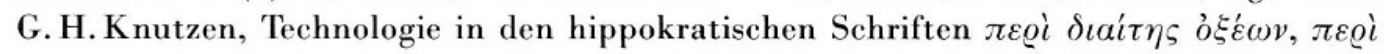


$\grave{\alpha} \gamma \mu \tilde{\omega} \nu, \pi \varepsilon \varrho \grave{\alpha} \varrho \vartheta^{\prime} \varrho \omega \nu \dot{\varepsilon} \mu \beta \circ \lambda \tilde{\eta} \varsigma$, Akad. d. Wiss. u. d. Lit., Abh. d. geistes- u. sozialwiss. Kl. 1963, Nr. 14, Wiesbaden 1964, S.34f.

14. Siehe H.Grensemann, a.a.O., S.53-64, und J.Jouanna, a.a.O., S.129-160 u. 508 Anm.1.

15 Siehe dazu ausführlich J. Kollesch, a.a. O., S.108-119, und G. Harig, Bemerkungen zum Verhältnis der griechischen zur altorientalischen Medizin, in: Corpus Hippocraticum (s. Anm. 9), S. 87-94.

16 Es sind dies die Beschreibung von Symptomen bei einer Nierenerkrankung und die Schilderung einer krankhaften Form der Harnausscheidung, die Rufus von Ephesos (De corp. hum. part. appellat. 190: S. 159,13-160,2 Daremberg-Ruelle) und Galen (In Hipp. Epid. VI comm. I 29: CMG V 10,2,2, S. 54,1-4) als wörtliche Zitate aus den «Knidischen Sentenzen» überliefert haben sowie die bereits genannte kritische Auseinandersetzung mit dieser Schrift zu Beginn von De diaeta acutorum (Kap.1-3. 5 [1. 2]: I, S. 109,2-110,13; 111,4-6 Kühlewein = II, S. 224,2-228,6; 232,5-7 Littré).

17 Zur möglichen Zuweisung von De diaeta und De septimanis an Euryphon s. Galen, De alim. fac. I 1,35: CMG V 4,2, S.212,18-20; In Hipp. De victu acut. comm. I 17: CMG V 9,1, S. 135,2-5; De diaeta in morb. acut. sec. Hipp. 9,3: CMG Suppl. Or.II, S. 109,17-21 (an der zuletzt genannten Stelle ist De septimanis, wie bisweilen auch sonst bei Galen, mit dem Titel «Die kleinere Abhandlung über die Krankheiten» zitiert).

18 Anonymi Londinensis ex Aristotelis iatricis Menoniis et alis medicis eclogae, hrsg. v. H. Diels, Berlin 1893 (Supplementum Aristotelicum III 1), IV 31-40 (im folgenden zitiert als Anon. Lond.).

19 Siehe Galen, De instrum. odorat. 2,3: CMG Suppl. V, S. 36,10f.

20 Galen, De caus. procataret. 192: CMG Suppl. II, S. 52,3-9.

21 Galen, In Hipp. Epid. VI comm. I 29: CMG V 10,2,2, S. 55, $10-16$.

22 Hippokrates, De morb. II 68: S.207,1-7 Jouanna = VII, S. 104,1-7 Littré.

23 Vgl. J. Kollesch, a. a. O., S.111.

24 Siehe Caelius Aurelianus, De morb. acut. II 96 f.; De morb. chron. II 121.

25 Vgl. H.Grensemann, a. a. O., S.36.

26 Siehe E.Littré, Euvres complètes d'Hippocrate, Bd.I, S.206f. u. 214; M. Wellmann, Art. «Euryphon», in: Paulys Real-Encyclopädie der classischen Altertumswissensehaft, hrsg. v. G. Wissowa, Bd. VI, Stuttgart 1909, Sp. 1343.

27 Siehe Caelius Aurelianus, De morb. chron. III 139; Galen, De bonis malisque suc. 4,28: CMG V 4,2, S.404,17-22; De marasmo 9: VII, S.701,8-13 Kühn; Meth.med.VII 6: X, S. 474, 14-475,2 Kühn; De venae sect. adv. Erasistr. 1: XI, S. 149, 12-150,6 Kühn; De simpl. med. temp. et fac. VI prooem.: XI, S. 795,8-15 Kühn; [Galen], De succed.: XIX, S. 721,3-5 Kühn.

28 Siehe M. Wellmann, a. a. 0 .

29 Siehe Censorinus, De die nat. 7,5.

30 M. Wellmann, a.a.O.

31 Siehe die Erläuterungen zu dieser Stelle von D. Nickel, CMG V 2,1, S. 89 f. zu S. 50,4-7.

32 Galen, De bonis malisque suc. 4,28: CMG V 4,2, S.404,17-22; De marasmo 9: VII, S. 701,8-13 Kühn; Meth. med. VII 6: X 474,14-475,2 Kühn.

33 Caelius Aurelianus, De morb. chron. III 139; Aetius Amidenus, Lib. med. X 29: M. Wellmann, Die pneumatische Schule bis auf Archigenes in ihrer Entwickelung darge- 
stellt, Berlin 1895 (Philologische Untersuchungen, hrsg. v. A. Kießling u. U. v. Wilamowitz-Moellendorff, H.14), S.59, Anm. 2 von S.55. Zu den Einzelheiten der Therapie s. S. 18.

34 H. Grensemann, a. a. O., S. 14 f.; vgl. auch S. 198-201.

35 Vgl. ebd., S. 198.

$36 \mathrm{Zu}$ den näheren Lebensumständen des Ktesias s. T.S. Brown, Suggestions for a vita of Ctesias of Cnidus, Historia 27 (1978), S.1-19.

37 W. Artelt, Studien zur Geschichte der Begriffe «Heilmittel» und «Gift». Urzeit - Homer Corpus Hippocraticum, Leipzig 1937 (Studien zur Geschichte d. Medizin, hrsg. v. K.Sudhoff, H.23), S. 78 f. (Nachdruck Darmstadt 1968).

38 H. Grensemann, a. a. O., S. 196.

39 Vgl. G. Harig, Anfänge der theoretischen Pharmakologie im Corpus Hippocraticum, in: Hippocratica. Actes du Colloque hippocratique de Paris (4-9 septembre 1978), hrsg. v. M.D.Grmek, Paris 1980 (Colloques internationaux du Centre National de la Recherche Scientifique, No.583), S.227-239.

40 Siehe Galen, In Hipp. De artic. comm. IV 40: XVIII A, S. 731,6-8 Kühn.

41 Hippokrates, De artic. 51-60 u. 70-78: II, S. 188,21-210,13 u. 224,18-239,6 Kühlewein $=$ IV, S. 224,6-260,8 u. 288,11-316,8 Littré.

42 Die Fragmente der Griechischen Historiker (FGrHist) 688 F 20, hrsg. v. F. Jacoby, III C, Leiden 1958, S. 475,29-476,13.

43 FGrHist 688 F 45: III C, S. 497,25f.

44. FGrHist 688 F 14: III C, S. 467,28-468,6. Zum Folgenden vgl. G. Harig und J. Kollesch, Der hippokratische Eid. Zur Entstehung der antiken medizinischen Deontologie, Philologus 122 (1978), S.174f.

45 Siehe Hippokrates, Iusiur. 6: CMG I 1, S. 5,1-4.

46 Zum Folgenden vgl. J. Kollesch, a. a. O., S.119-122.

47 R. Joly, Le système cnidien des humeurs, in: La Collection hippocratique et son rôle dans l'histoire de la médecine. Colloque de Strasbourg (23-27 octobre 1972), hrsg. v. L. Bourgey und J. Jouanna, Leiden 1975 (Université des Sciences Humaines de Strasbourg, Travaux du Centre de Recherche sur le Proche-Orient et la Grèce Antiques 2), S. 107-127.

\section{Summary}

\section{Cnidus as a centre of early scientific medicine in ancient Greece}

In the fifth century B.C., Cnidus and Cos, geographical neighbours and closely related also through the family of the Asclepiads, created an homogeneous though varied medicine. Available sources do not allow us - contrary to what is often done - to state a principal difference between Cnidian and Coan medicine. The Cnidian physicians Euryphon, Herodicus and Ctesias are presented. 


\section{Résumé}

Cnide comme centre de la médecine scientifique primitive dans la Grèce antique

Proches géographiquement et en relation à travers la famille des Asclépiades, Cos et Cnide constituent au $5^{e}$ siècle av. J.-C. une médecine qui, tout en étant diverse, forme une unité. Létat de nos sources ne nous permet pas, comme cela a été fait souvent, d'établir une différence fondamentale entre médecine cnidienne et médecine coaque. Présentation des médecins cnidiens Euryphon, Hérodicos et Ctésias.

Dr. sc. Jutta Kollesch

Akademie der Wissenschaften der DDR

Zentralinstitut für Alte Geschichte und Archäologie

Leipziger Straße 3-4.

DDR-1086 Berlin 